\title{
A parsimonious laboratory system for the evaluation of rat reaching task: recovery from the massive destruction of motor areas
}

\author{
Youki Manaka $^{1,2}$, Yoichi Ohno ${ }^{1,3, *}$, Akinori Horikoshi ${ }^{1,4}$, Kazuyuki Imamura ${ }^{1}$ \\ ${ }^{1}$ Department of Systems Life Engineering, Maebashi Institute of Technology, Kamisadori-machi, 371-0816 Maebashi, Gunma, Japan \\ ${ }^{2}$ Department of Technological ME Research and Development, TOP Co. Ltd., 120-0035 Tokyo, Japan \\ ${ }^{3}$ Department of Physical Therapy, Faculty of Health Care, Takasaki University of Health and Welfare, Nakaoruicho, 370-0033 Takasaki, Gunma, Japan \\ ${ }^{4}$ Accounting Affairs, Ono Sokki, Co. Ltd., 222-8507 Yokohama, Japan \\ *Correspondence: ohno@takasaki-u.ac.jp (Yoichi Ohno)
}

DOI:10.31083/j.jin2004096

This is an open access article under the CC BY 4.0 license (https://creativecommons.org/licenses/by/4.0/).

Submitted: 16 August 2021 Revised: 25 November 2021 Accepted: 30 November 2021 Published: 30 December 2021

The rat reaching task is one of the best paradigms from behavioral study of upper limb movements. Rats are trained to reach and grab a pellet by extending their hand through a vertical slit. A few conventional imaging systems specific for the rat reaching task are commercially available with a high installation cost. Based on image analysis of video recordings obtained during the reaching task, we, herewith, developed a new, low-cost laboratory system that can be used for the quantitative analysis of ten basic forearm movements, in contrast to subjective assessments used in previous studies. We quantified images of the pronated and supinated palm and the accuracy and speed of reaching the target. Applying this newly developed method, we compared the forearm movements during the reaching task before and after a massive anatomical lesion of the sensorimotor cortex performed by tissue aspiration. We also wanted to investigate the recovery of upper limb function possibly induced by repeating the task for a relatively short term of a few weeks. In the experiment, 7 injured groups and 3 control groups were used. We found characteristic abnormalities of the forearm movements and a significant recovery in the success rate of grasping the target pellet. The present results demonstrate that our method is straightforward for the quantitative evaluation of forearm movements during the reaching task primarily controlled by the sensorimotor cortex.

Keywords

Reaching task; Forearm movement; Video image analysis; Sensorimotor cortex

\section{Introduction}

Stroke sequelae lead to reduced quality of life [1]. A majority $(80 \%)$ of patients with stroke exhibit some difficulty in forearm movements [2], and $60 \%$ chronically suffer from movement disabilities that affect their daily life [3]. It is also known that muscular strength and delicacy are impaired when the motor cortex is impaired in addition to motor paralysis $[4,5]$. Upper limb and forearm motility form the basis of movements involved in daily activities, such as touching, grabbing, and moving objects. These upper limb motions include a complex finger, palm, wrist, elbow, and shoulder movements. After a stroke, it is often difficult to re- gain the ability to perform complex upper limb movements [6-8]. On the other hand, it has been reported that active use of the injured upper limb by rehabilitation activates the activity of the motor cortex and recovers the movement disorder $[9,10]$.

The rat reaching task (RT) has been used to study upper limb function because the underpinning anatomy is similar between rats, primates, and humans [11-13]. Rats performing the RT extend their preferred hands through a narrow slit toward the target of a single pellet placed on a shelf in a reaching box. The rat can eat the pellet after withdrawing the hand with it through the slit. Some training is necessary to accomplish this skilled reaching. The ten elements of movements for the RT are described in detail in section 2.2 in the materials and methods [14-16].

In previous studies, videos of the RT were recorded, and the elements of hand reaching were subjectively analyzed by individually reviewing the pertinent images [17-20]. The analysis was mainly based on the success rate of the task under various scoring schemes. In the present study, we developed new software for analyzing video images of the ten RT elements that involve the simultaneous identification of different pre-colored fingers in action. Our method enables the quantitative evaluation of all aspects of hand movements in the RT and makes a straightforward comparison between groups of animals. It requires no expensive equipment. To demonstrate the applicability of our method for longitudinal observations, we examined the effects of large cortical ablation and rehabilitation, including the sensorimotor cortex, on the RT through repeated measurements obtained before and at several time points after the experimental lesion.

\section{Materials and methods}

2.1 Animals

The present study used ten adult Long-Evans rats of both sexes (body weight: $300-400 \mathrm{~g}$ ). They were separately 
housed in standard cages and kept in the experiment room in $12 / 12 \mathrm{~h}$ light/dark conditions. Seven of the ten rats were included in the cortical lesion group, and the remaining three were included in the sham-operated control group. During the experiments, food deprivation was performed to maintain a bodyweight of $90-95 \%$ of the initial body weight. Water was available ad libitum.

A 5-mm thick, custom-made acrylic plastic box was used for the RT. Its size was $450 \times 250 \times 300 \mathrm{~mm}$ (length $\times$ width $\times$ height). A vertical slit of $10 \times 150 \mathrm{~mm}$ was carved $30 \mathrm{~mm}$ from the floor at one end of the box. A 40-mm-wide shelf was attached at the bottom of the slit outside the box. In front of the slit, there was a hollow designed to hold a single pellet (45 mg, \#F0021, Bioserve Inc., NJ, USA).

\subsection{Motor elements of the RT}

For the RT, the rat's nose is placed into the slit, and both its forearms are placed on the floor (Fig. 1A). After noticing a pellet on the shelf, the rat raises its dominant hand toward the midline (Element 1, Fig. 1A). The hand is partially closed while passing through the slit toward the pellet (Element 2, Fig. 1B). The elbow moves to the midline with partial pronation (Element 3, Fig. 1B), and the forearm extends toward the target through the slit (Element 4, Fig. 1C). Opening the hand widely (Element 5, Fig. 1D), the rat touches the pellet after further pronating the forearm (Element 6, Fig. 1E). After grabbing the pellet (Element 7, Fig. $1 \mathrm{~F}$ ), a supination of approximately $90^{\circ}$ is performed (Element 8 , Fig. $1 \mathrm{G}$ ) in preparation for withdrawing the forearm through the slit. The rat shows further supination of approximately $45^{\circ}$ (Element 9 , Fig. $1 \mathrm{H})$ to carry the pellet toward the mouth. Finally, the hand is opened to release the pellet into the oral cavity (Element 10, Fig. 1I). Accordingly, we could evaluate all ten elements of forearm movement during RT.

\section{$2.3 R T$ training}

The rat was first acclimated to the test box with pellets scattered on the box floor. The training began with a few pellets placed on the shelf close to the slit so that the trainee could reach them with the tongue. Subsequently, the pellets were placed at a gradually increasing distance from the slit to stimulate the rat to retrieve the pellet by hand. The RT training started after determining the dominant hand and continued until the rats consistently obtained the pellet with a success rate of $70 \%$ or more in 20 consecutive trials. There is no difference between the success rate before painting and the success rate immediately after painting in all individuals, and it has been confirmed that painting on the finger does not affect RT. The criterion for success was that the forelimb passed through the slit in the anterior wall, grasped the pellet, and passed through the slit again to bring the pellet to the mouth.

\subsection{Finger painting}

Under anesthesia with 1-2\% isoflurane in a gas mixture of $\mathrm{N}_{2} \mathrm{O}: \mathrm{O}_{2}=2: 1$, the first and fifth fingers of well-trained rats were coated with paints of different colors (blue and green or yellow; car paint, Musashi Holt Corp., Tokyo, Japan). The paint was dried, the rat emerged from anesthesia, and after at least an hour, the rat was placed in an RT box for continuous recordings of their upper limb movements from two different view angles using two digital cameras (EX-ZR400, and ZR200 CASIO COMPUTER Co., Ltd, Tokyo, Japan) in high-speed mode ( 240 frames/sec, $512 \times 384$ pixels) with the settings described in section 2.5 .

\subsection{High-speed photography and image analysis}

The forearm movements of the rats were recorded in two orthogonal directions, the frontal and bottom views. A small light-emitting diode that switched on and off at a rate of $1 \mathrm{~Hz}$ was placed in the field of view of both cameras to synchronize them. The two image series were manually synchronized in a frame-by-frame manner using the light-emitting diode lighting as a reference.

First, the blue and green (or yellow) regions painted on the fingertips were automatically extracted from the images and labeled. After fitting ellipses on the color-extracted areas, the outline of each finger was determined. Subsequently, the ellipse's long (blue) and short (green) axes were determined, and the endpoint of the long axis was considered the fingertip. The value of the angle between the line connecting the first and fifth fingertips and the horizontal line of the food shelf was calculated (Fig. 2).

We measured the following parameters from the frontalview images, (1) the distance between the first and fifth fingertips, and (2) the angle between the line connecting the two fingertips and the horizontal line of the shelf (these two measures were automatically extracted using custom-made software (Microsoft Visual C++ 2010, Microsoft Inc., Washington, DC, USA), including the Open CV library (Intel 2.4, Intel Inc., CA, USA)); we visually confirmed that the custom-made program automatically captured the various aspects of the RT from high-speed videos. Fig. 2 shows images of pronation (A) and supination (B) to demonstrate the appropriateness of the current scheme for measuring them.

From the bottom-view images, we measured (3) the distance from each of the five fingertips to the center of the pellet when the dominant hand touched it, and (4) the hand-closing velocity when the pellet was grabbed. The images analyzed were trimmed and converted to binary images (Fig. 3A). After noise reduction, their labels were processed to linearize the border of the hand. Next, by placing small dots on this borderline, the fingertips were determined (Fig. 3B). More precisely, when the angle between three successive dots on the line was below $115^{\circ}$, the center point was determined to be the fingertip (Fig. 3C).

An ellipse was fitted on the post-pellet-retrieval image, with the center placed at the center of the pellet. Subsequently, we determined the distance between each fingertip and the center of the pellet.

To analyze all motor elements of the RT, the motion analysis targets were only the trials that succeeded by 20 RT trials. 


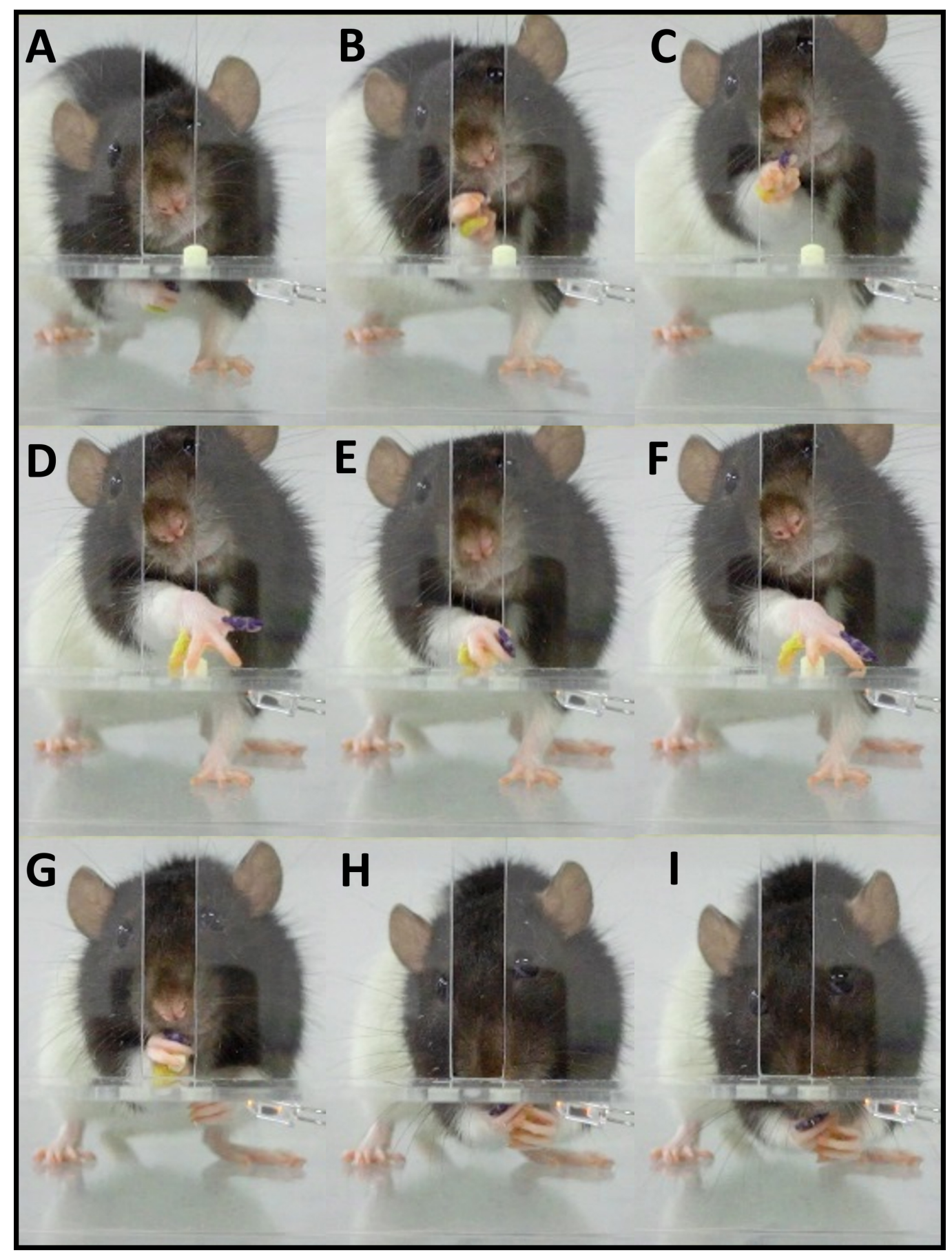

Fig. 1. Motor elements of the reaching task (RT). (A) Fingers to midline (Element 1). (B) Fingers semiflexed (Element 2) and Elbow to midline (Element 3). (C) Advance (Element 4). (D) Fingers extend (Element 5). (E) Pronation (Element 6). (F) Grab (Element 7). (G) Supination 1 (Element 8). (H) Supination 2 (Element 9). (I) Release (Element 10).

\subsection{Ablation of the sensorimotor cortex}

In the three sham-operated rats, a small piece of the skull was removed from the frontal area of the head, leaving almost no trace of damage to the cortex; in the seven rats of the experimental group, a large amount of cortical tissue was removed. In these rats, a large area of the frontal cortex, including the sensorimotor region (the rostral forelimb motor area and caudal forelimb motor area), was removed by tissue aspiration under general anesthesia (ketamine $75 \mathrm{mg} / \mathrm{kg}$, Xylazine $7.5 \mathrm{mg} / \mathrm{kg}$, intraperitoneally) in the hemisphere contralateral to the rat's dominant hand (Fig. 4).

Coagulation was performed for blood stanching during aspiration. The empty space thus created was filled with an absorbable gelatin sponge (Spongel, LTL pharma Co., Tokyo, Japan), and the scalp was sutured with nylon thread. The suture site was disinfected with benzalkonium chloride $(1.0 \%$ 


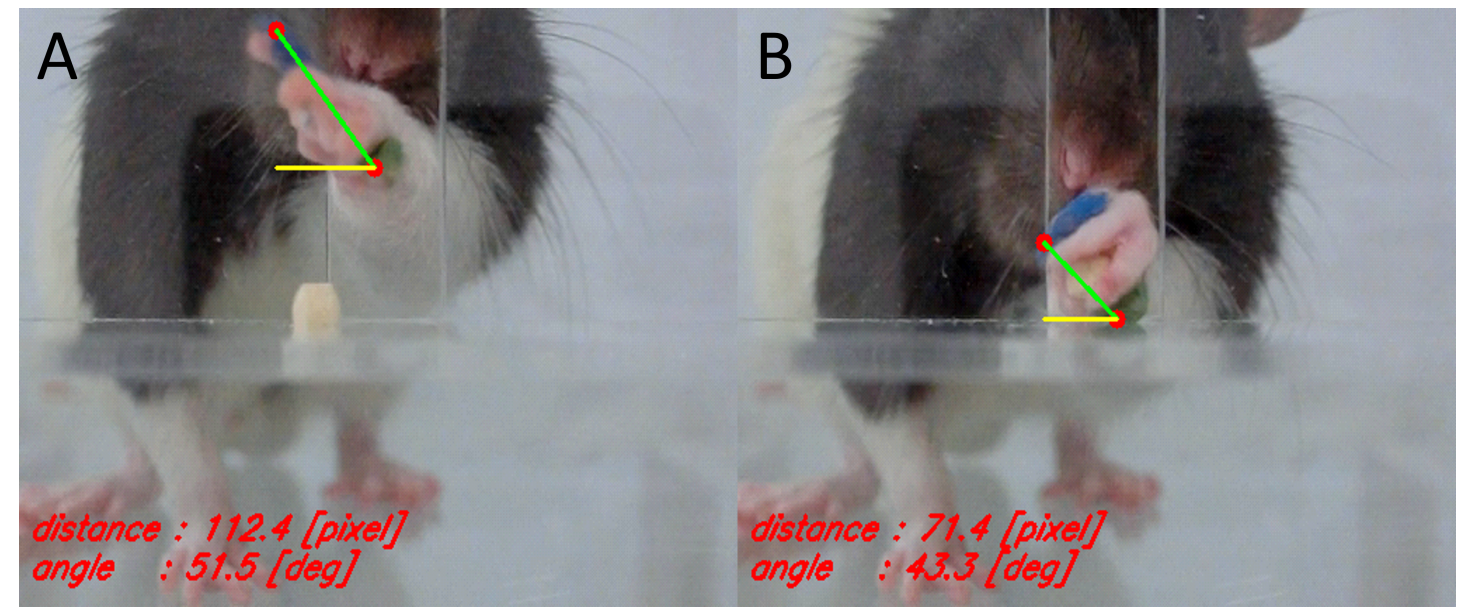

Fig. 2. Frontal-view image analysis. The tip of the first and fifth fingers (red dots) is determined by fitting an ellipse onto each finger's extracted color (blue) images. Images of pronation (A) and supination (B) measure the distance between the first and fifth fingertips as indicated by the green lines. The angle between the fingertip line and the frontal shelf horizontal line is also analyzed (angles depicted between yellow and green lines). These values are automatically displayed at the bottom corner of each image.
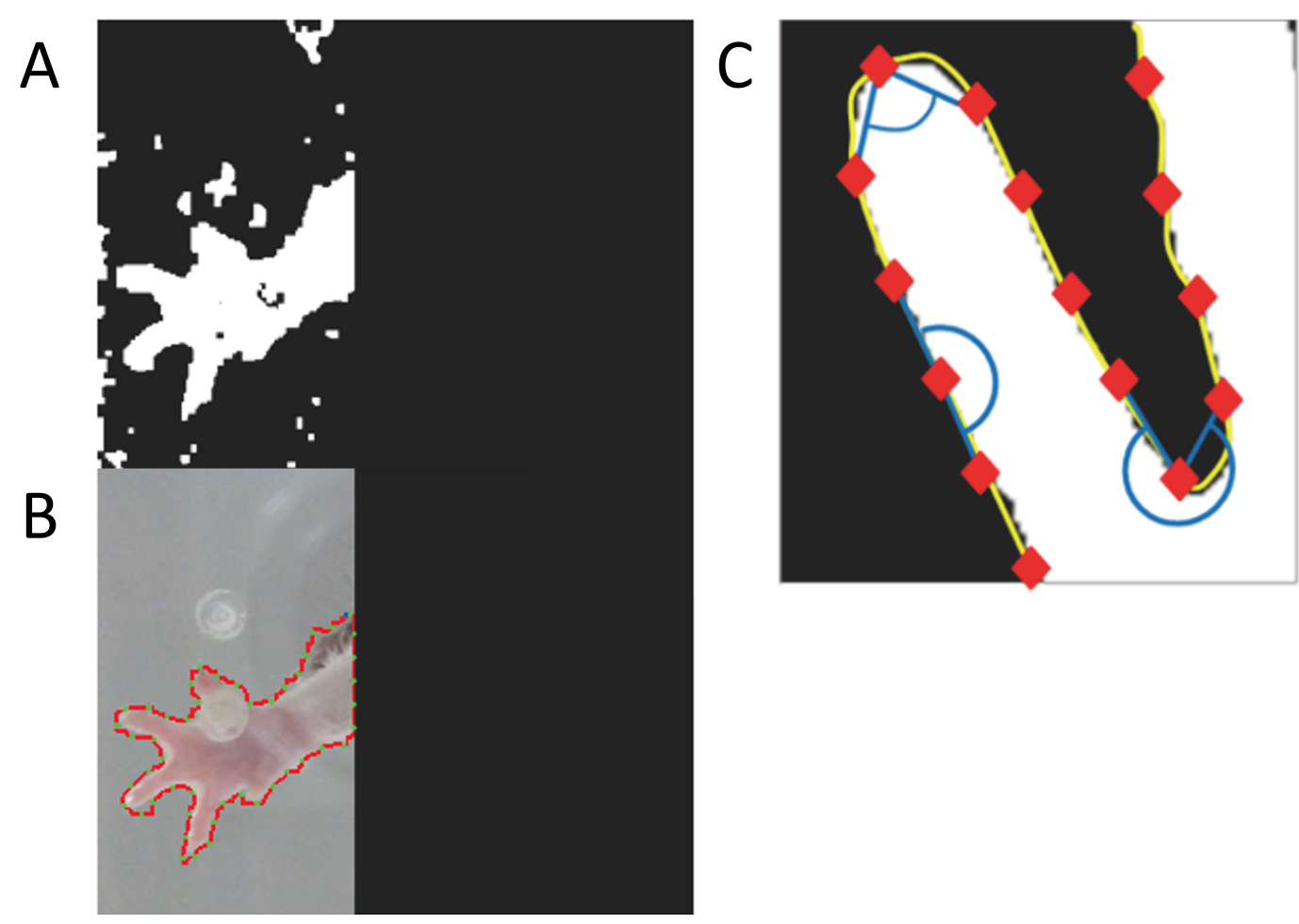

Fig. 3. Bottom-view image analysis. The bottom-view images obtained at the time of contact between the hand and the pellet are used to identify the tip of each finger. A binarized image of the hand (white area in A) is obtained against the background (black) using a background-differencing technique. After removing the noise and applying the labeling process, the hand outline is extracted (red line in B). Dots are placed on the contour lines (green dots on B and red diamonds in $\mathrm{C}$ ), and the tip of each finger is considered as the point at which a sharp angle $\left(<115^{\circ}\right)$ is made by three successive points $($ blue angles in $\mathrm{C}$ ).

solution of benzalkonium chloride, ${ }^{\circledR}$ Osban, Nihon Pharmaceutical Co. Ltd., Tokyo, Japan) twice a day and intramuscular antibiotic injections were administered daily for seven days (ampicillin sodium, Viccillin ${ }^{\circledR}$, Meiji Holdings Co., Ltd, Tokyo, Japan).

In some cases, after the end of all experiments, we performed intra-cardiac perfusion of $4 \%$ paraformaldehyde so- lution under deep anesthesia. The brain was removed for the following histological examination with the Nissl-staining.

\subsection{RT resumption}

After a recovery period of three days, the rats performed $20 \mathrm{RT}$ trials every day from the 4th to the 17 th day after the ablation. The rate of successful pellet retrieval was measured every day during the recovery period of two weeks. We 
A
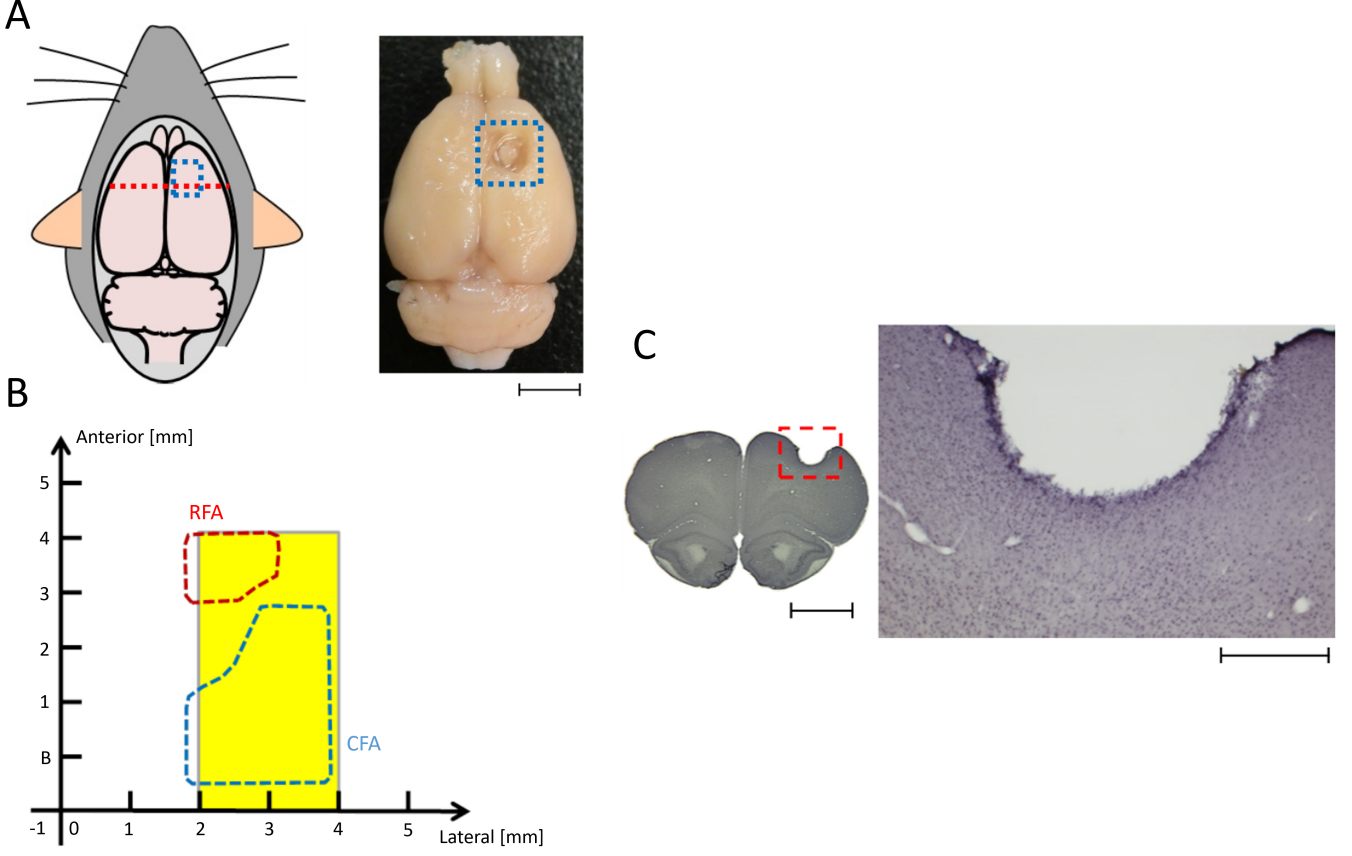

Fig. 4. Schematic diagram of the damaged area and the state of the damage. (A) It is a rat brain from above. The red dotted line in the figure indicates the position of the bregma. The blue dotted line in the figure was damaged area. The scale bars indicate $5 \mathrm{~mm}$. (B) It is an enlarged view of the square part of the blue dotted line in the figure of (A). The vertical axis is 0 for bregma (B) and positive for the anterior, and the horizontal axis is 0 for the median and positive for the outer (Lateral). The forelimb motor cortex of rats has Rostral Forelimb Area (RFA) and Caudal Forelimb Area (CFA). The damage this time was set to the yellow area in the figure surrounding these two areas. (C) A Nissl stained section shows the lesion induced by the cortical aspiration. A part with a red rectangle was enlarged to the right. The scale bars indicate $2 \mathrm{~mm}$ and $500 \mu \mathrm{m}$, respectively.

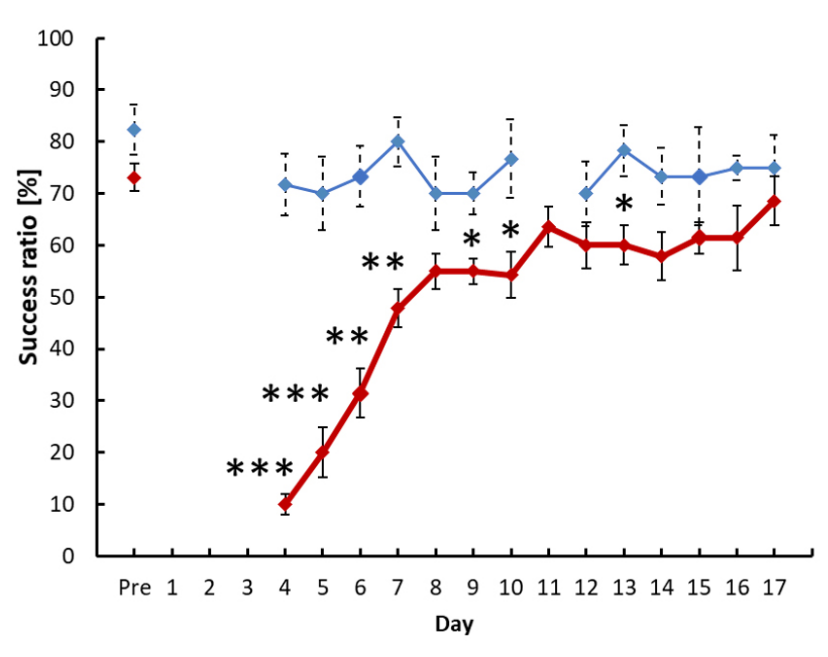

Fig. 5. Changes in the reaching task (RT) success rate. The RT success rate (\%) following the cortical lesion is shown. The red line represents the lesioned rats $(N=7)$, while the blue line the sham-operated rats $(N=3)$. Before the ablation, both groups of rats performed the test with a success rate of $>70 \% .{ }^{* * *} p<0.001,{ }^{* *} p<0.01,{ }^{*} p<0.05$ (Student's $t$-test). Data are represented as mean \pm SEM.

have compared RT performance at the 6th and 15th days after the cortical lesion. Previously, there were various reports on the recovery period $[18,21,22]$. Shorter ones were reported to have no reduction to RT performance after the 8th post-lesion [23]. For that reason, we have set the first measurements on the 6th day with an additional practical reason for the surgical aftereffects. The second focusing point was set on the 15th day. This is based on the previous studies, most of which had measured for two weeks after the lesion $[18,22,24]$. And the rate of successful pellet retrieval in RTs (i.e., percentage of successful trials in 20 challenges) was examined at different time points after the cortical ablation and compared with the average rate from five successive daily trials performed before the ablation.

\subsection{Histological examination}

The lesion extent was histologically evaluated after the termination of the behavioral studies to ensure the presence of similar cortical lesions in the sensorimotor areas of all experimental rats.

Upon termination of all experiments, the rats were deeply anesthetized and sacrificed with sodium pentobarbital (100 $\mathrm{mg} / \mathrm{kg}$, intraperitoneal), immediately followed by transcardial perfusion of saline, followed by $4 \%$ paraformaldehyde administration. After cryoprotection of the removed brain tissue, $50-\mu \mathrm{m}$-thick coronal sections were cut using a freezing microtome. The thin sections were mounted on glass slides and stained with thionine. Finally, the damaged cortical area was histologically evaluated with direct vision and microscopy. 


\subsection{Statistical analysis}

Measurements are presented as means and standard errors of the means (mean \pm SEM). Student's $t$-test and twoway analysis of variance (ANOVA) were used to evaluate the RT success rate. The student's $t$-test and Tukey-Kramer's test were used to evaluate the other variables (fingertip distance, fingertip angle, hand-closing velocity) at two time points. Tukey-Kramer's test were used to evaluate the distance from each of the five fingertips to the pellet. SPSS for Windows (ver. 17.0, IBM, New York, USA) was used for all statistical analyses, and the significance level was set at $5 \%$.

\section{Results \\ 3.1 Decrease in the RT success rate following cortical ablation}

The change after the ablation success rate was not significantly changed in sham-operated rats (ANOVA, F $(13,28)$ $=0.1, p>0.05)$, but was significantly increased in lesioned rats $($ ANOVA, $F(13,84)=15.1, p<0.001)$. The lowest rate of successful food retrieval in the $\mathrm{RT}$ was observed on the 4th day after the ablation (Fig. 5, red line; blue lines for sham-operated rats). The lowest success rate was observed on the 4th day after the ablation (the first post-ablation measurement). Subsequently, the rate steadily increased until the 8th day. Although the pace decreased, further recovery was obtained until the 17th day, when the success rate was similar to or slightly more than the pre-ablation rate. A significant difference was also noted between cortex-ablated rats and sham-operated rats (two-way ANOVA, F $(1,112)=125.3$, $p<0.001)$. A significant difference was observed between the two groups on the 4th, 5th, 6th, 7th, 9th, 10th, and 13 th days after the operation (Student $t$-test, $p<0.05$ ).

\subsection{Changes in the two-fingertip distance and pronation angles}

The distance between the first and fifth fingertips and the angle between the line connecting the two fingertips and the horizontal line of the food shelf was measured at threetime points: before, 6th days after, and 15th days after the cortical ablation (Fig. 6). Before the ablation, the hand was raised from the floor $0.6 \mathrm{~s}$ before grabbing the pellet. The fingertip separation was found to increase shortly (approximately $0.3 \mathrm{~s}$ ) before grabbing the pellet (Fig. 6, time zero), with a maximal separation of $20 \mathrm{~mm}$ (Fig. 6, green line). We noted no significant difference among the three-time points in the maximal separation between the first and fifth fingertips (Fig. 7A), indicating that the cortical lesion did not significantly affect the maximal extent of the separation between the first and fifth fingertip in the RT behavior. On the other hand, a comparison between the lesioned rats and sham-operated rats (Fig. 7C) showed that sham-operated rats were significantly smaller 15 th days after (Student's $t$-test, $p$ $<0.05)$

On the other hand, the pronation angle was strongly affected by the pre-lesion. In the pre-lesion, the pronation angle decreased shortly before grabbing the pellet and increased to $160^{\circ}$ after that (Fig. $6 \mathrm{~A}$, orange line). 6th day after the cortical ablation, the hand left the floor $0.7 \mathrm{~s}$ before grabbing the pellet, and the angle increased $0.4 \mathrm{~s}$ before grabbing the pellet. The angle decreased immediately before the hand gripped the pellet. Moreover, the pronation angle changed slowly, with an angle of less than $50 \%$ of the angle of the pre-lesion (Fig. 6B). After 15th days, the hand was raised from the floor $0.6 \mathrm{~s}$ before grabbing the pellet, and the angle decreased $0.3 \mathrm{~s}$ before grabbing the pellet. Subsequently, a gradual increase in the angle was observed, which was smaller than in prelesion; however, it was greater than the angle on the 6th day after the ablation.

The maximum pronation angle was found to be significantly reduced both 6 th and 15 th days after the lesion (TukeyKramer's test, $p<0.01$ ), with a trend toward recovery by the 15 th day (Fig. 8A). However, despite the trend toward recovery in the maximum angle, the pronation movement was notably slower on the 6 th (Fig. 6B, orange line) and 15th (Fig. 6C, orange line) days after the lesion. In addition, a comparison between the lesioned rats and sham-operated rats (Fig. 8C) revealed that the sham-operated rats exhibited significantly larger values at the post-lesion days 6th (Student's $t$-test, $p<0.01$ ) and 15th (Student's $t$-test, $p<0.05$ ).

\subsection{Changes in the grabbing speed}

We also examined the speed of hand movement from the moment of maximum hand opening to the moment the pellet was grabbed at the three-time points (pre-lesion, 6th days after, and 15th days after). We divided the time spent for handclosing into three equally long phases: the initial, middle, and final phases (purple, initial; orange middle; and green, final). In the three phases, the time required to grip the pellet from the time when the distance between the first and fifth fingers shows the maximum value is divided into 3 (33.3\%). Then, the time spent in the three phases at each point was compared (Fig. 9).

In pre-lesion, we found that the hand-closing speed steadily increased once the action was set in motion (TukeyKramer's test, $p<0.05$ ). The cortical lesion significantly interfered with the acceleration process. After 15th days, there was a trend of increasing speed, suggesting partial recovery. There was no significant difference between lesioned rats and sham-operated rats at each time and phase.

\subsection{Spatial errors in targeting}

We measured the accuracy of the hand placement when the rat touched the pellet. The results were grouped by sex due to the innate difference in body size between male and female rats. The separation between each fingertip and the center of the pellet is shown for the male rats in Fig. 10A. 6th days after the lesion, the hand position differed from baseline: all five fingertips moved $5 \mathrm{~mm}$ forward from the respective loci in the pre-lesion. Compared to the pre-lesion and 15th days after ablation, the lateral hand shift was most visible for the third and fourth fingertips; the deviation was significant (Tukey-Kramer's test, $p<0.05$ ). The female rats had smaller hands, and there was no significant shift in the location of each fingertip upon touching the pellet (Fig. 10B). 

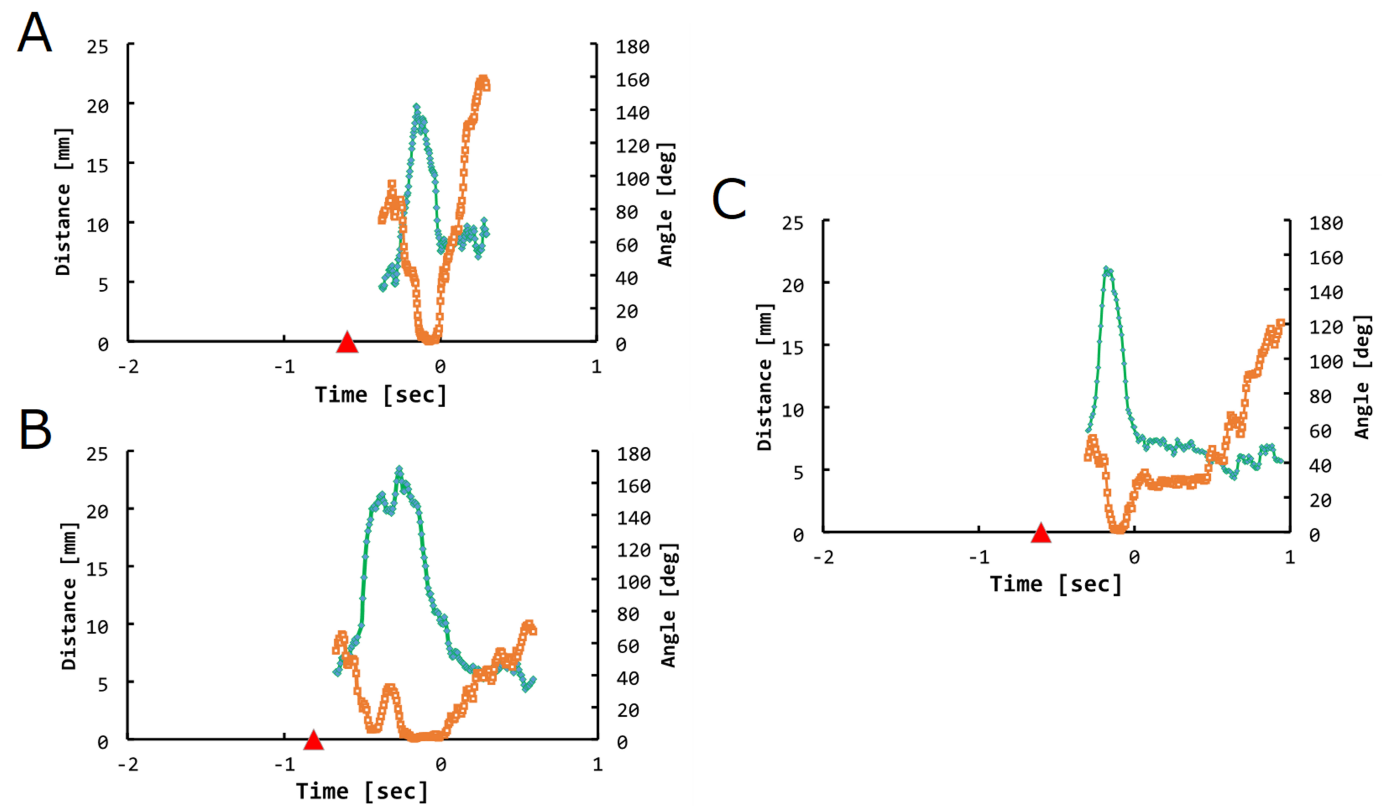

Fig. 6. Changes in the two-fingertip distance and pronation angle from a representative subject. The distance between the first and fifth fingertips (green) and the angles between the two-fingertip line and the horizontal line in pronation and supination are plotted before (A), 6th days after (B), and 15th days after (C) the cortical lesion. Representative pictures for pronation and supination are shown in Fig. 2A,B, respectively. Representative data are shown in this graph. The time zero on the $\mathrm{x}$-axis indicates the instant when the rat grabbed the pellet. The left and right halves of the orange curves indicate pronation and supination, respectively. The green triangles on the $\mathrm{x}$-axis indicate the instants when the dominant hand left the floor.
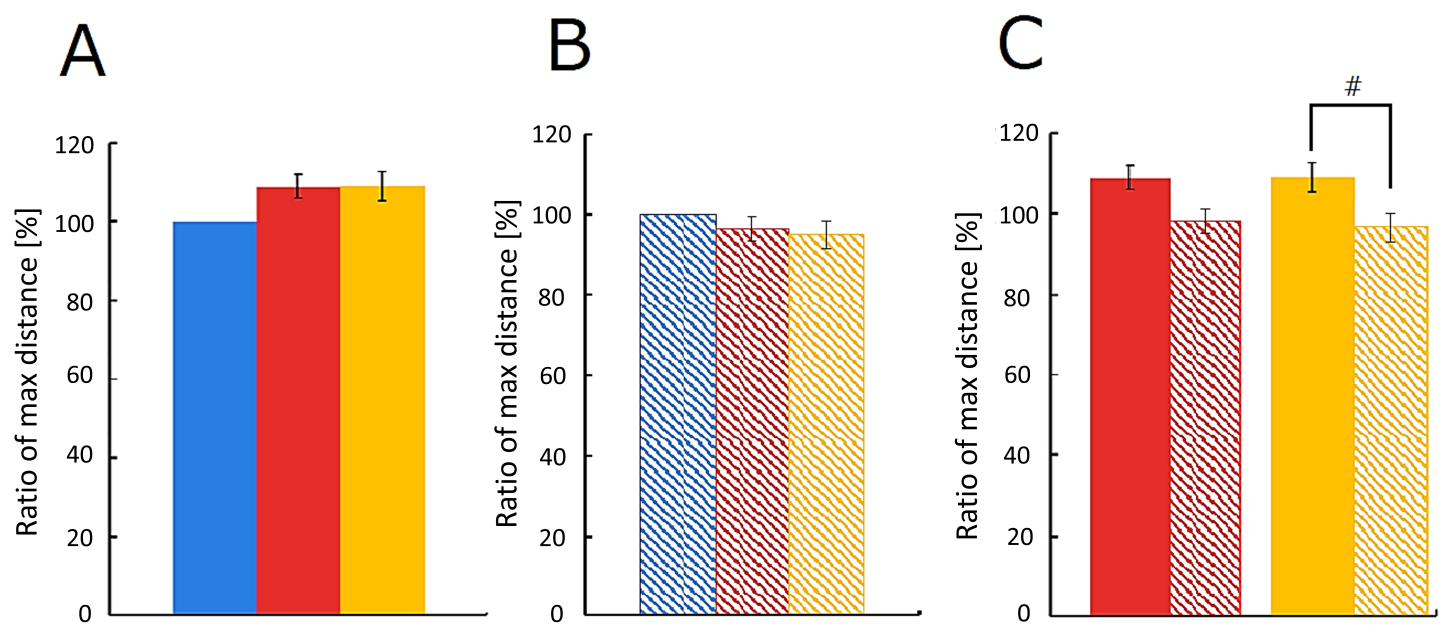

Fig. 7. Comparison of maximum distance. The lesion-induced changes are shown as percentages of the value before the lesion (blue bar). The maximum distance between the first and fifth fingertips in the lesioned rats (A) is compared before (blue column), 6th days after (red column), and 15th days after (yellow column) the cortical lesion. The data from sham-operated rats are shown in B. The maximum distance of each exhibits a small decrease with no statistical significance. A comparison between the lesioned rats and sham-operated rats (C) showed that sham-operated rats were significantly smaller 15 th days after (Student's $t$-test, \# $p<0.05)$. Data are represented as mean \pm SEM.

We found no significant difference in the average distance between the center of the pellet and the tips of each of the five fingers after the cortical lesion.

\section{Discussion}

Using the customized software we developed for image analysis, we meticulously studied the rats' hand movements during the RTs. Currently, the three-dimensional limb movements of rodents can be measured without using markers with DeepLabCut (EPFL Inc., VD, Suisse) [25]. However, to use DeepLabCut, a high-performance personal computer was needed. In contrast, our method enables precise RT analysis without requiring an expensive video-analysis system.

Since RT reported that the size of the injury affects the movement of the initial forelimbs, the injured area was set as large as possible in this study [26]. Extensive lesions involv- 

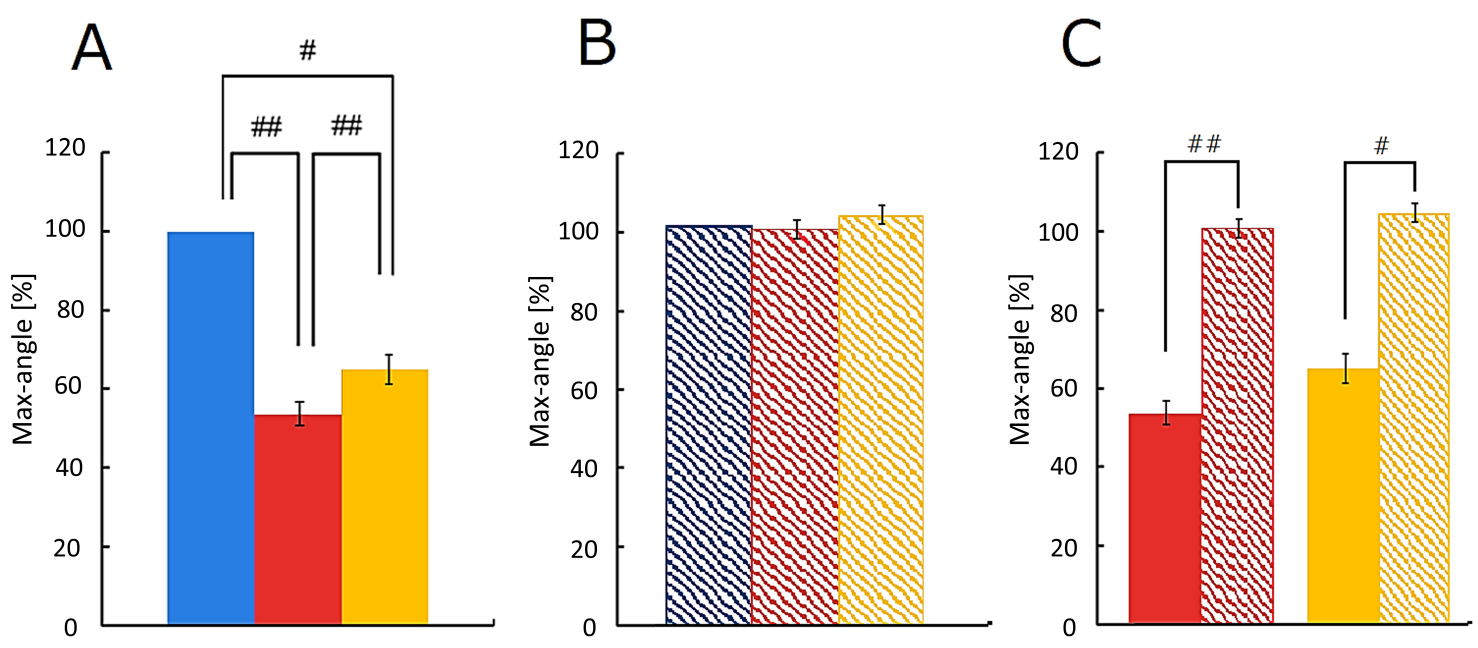

Fig. 8. Comparison of maximum pronation angle. The lesion-induced changes are shown as percentages of the value before the lesion (blue bar). The maximum pronation angle in the lesioned rats (A) is compared before (blue column), 6th days after (red column), and 15th days after (yellow column) the cortical lesion. The data from the sham-operated rats is shown in B. The lesioned rats significantly decreased in the 6th day after and 15 th days after the cortical lesion (Tukey-Kramer's test, \# $p<0.05$, \#\# $p<0.01$ ). A comparison between lesioned and sham-operated rats is shown in $(\mathrm{C})$. The sham-operated rats exhibited significantly bigger values 6th and 15th days after (Student's $t$-test, \# $p<0.05$, \#\# $p<0.01$ ). Data are represented as mean \pm SEM.

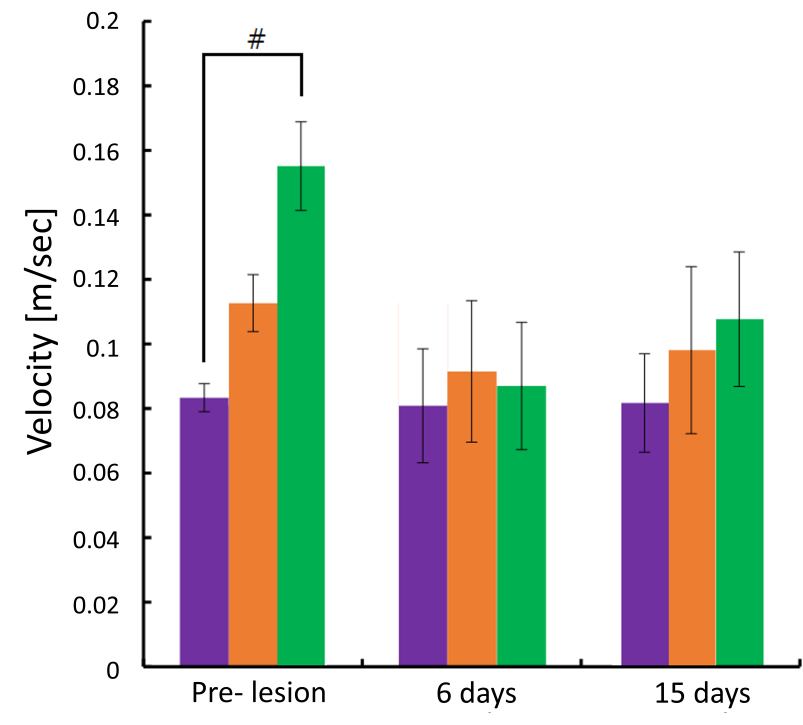

Fig. 9. Changes in hand movement speed. The time from maximal finger opening to pellet grabbing is divided into three equally long phases: the initial, middle, and final phase of the hand movement (purple, initial; orange middle; and green, final). The hand-closing velocity significantly increases from the initial to the final phase before the lesion (Tukey-Kramer's test, \# $p<0.05)$. However, this acceleration is almost completely suppressed after the cortical lesion. Data are represented as mean \pm SEM.

ing the sensorimotor cortex were found to reduce the RT success rate, which is consistent with previous reports $[17,18]$. However, the success rate reduction was found to be transient, occurring at four days after the ablation (the first day of postoperative tests) and rapidly recovering between the 4th and 8th day after the lesion, followed by a gradual increase to the control level on the 17 th day (Fig. 5). It has been re- ported that the recovery of forelimb function in rats is relatively early and spontaneous recovery $[27,28]$. It has also been reported that alternative exercise strategies are used as a factor as a reason $[18,24,29]$.

On the other hand, there is a report that the success rate of RT 14 days after motor cortex injury was 25\% [23]. Also, some studies reported an extraordinarily high level of brain plasticity following experimental perturbation [30, 31]. The effect of more active exercise has been reported to activate and compensate for the cerebral cortex $[9,10]$ and brain function on the non-impaired side [32]. Therefore, this may be due to the promotion of compensatory processes, including motor cortical plasticity, through repeated performance of the RT during the recovery period.

However, we also noted a semi-permanent loss of motor function. In the pronation angle analysis shown in Fig. 6 (the red curves in the negative area), we found the lesioned rats showed sustained difficulty in pronation. Additionally, they could not produce a supination movement after grabbing the pellet. The latter finding confirms what has been reported in previous studies $[33,34]$. These results suggest that the lesion produced in the present study was extensive enough to induce the retardation of neural mechanisms involved in multiple bodily movements. However, pronation recovered 15 th days after the cortical lesion (Fig. 8A), although supination remained greatly affected.

We found that hand opening speed and acceleration for grabbing the pellet decreased during the transition from the initial to the middle and from the middle to the final phase (Fig. 9). A motor cortical lesion has been reported to slow flexion movements and thus reduce grasping power [35]. Consistently with this report, our findings suggest that the speed reduction in the middle and final phases results from 
A

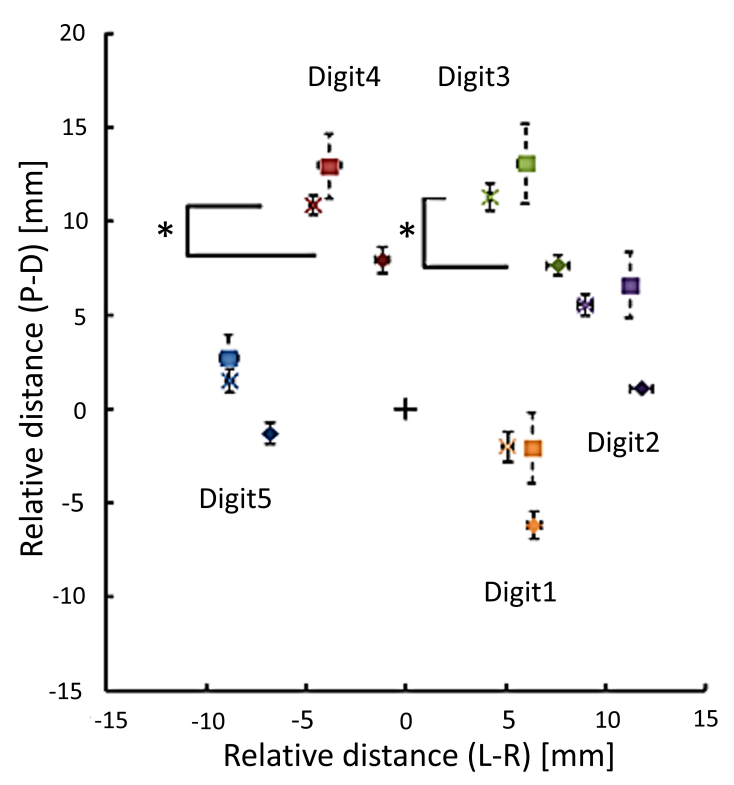

B

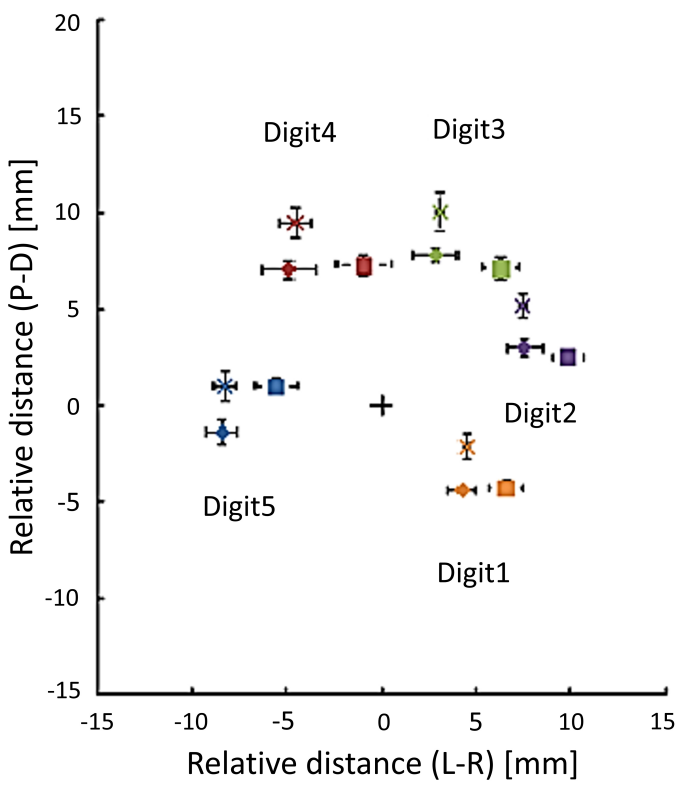

Fig. 10. The separation between each fingertip and the center of the pellet. Variation is seen in the position of each fingertip when the rats touch the pellet during the RT (A: males, B: females). The fingertips of each digit are shown in different colors. The + mark at the center $(0,0)$ indicates the center of the pellet (the point of origin). The animal is left-handed. The positive numbers in the $\mathrm{x}$-axis show the right direction and, in the $\mathrm{y}$-axis, the distal direction. Diamonds $(\checkmark)$ indicate the pre-lesion values, and squares $(\boldsymbol{\square})$ and crosses $(\otimes)$ indicate the values at the 6 th and 15 th days after the cortical lesion, respectively. Data are represented as mean \pm SEM.

the loss of cortical neurons involved in hand opening. This element of hand movement was not regained during the 15day recovery period.

In this study, the deviation of each fingertip from the target center was minimal in control conditions (i.e., less than $1.0 \mathrm{~mm}$ ). The cortical lesion significantly increased this placement error; the deviation involved all fingers (Fig. 10A). This may be due to errors in forearm and shoulder movements. The deterioration was not fully ameliorated following repeated RT performance during the recovery period, consistent with clinical observations [36]. The present study demonstrates that the quantitative analysis method we have developed may play a critical role in evaluating the efficiency and dependability of new treatments for hand movement disorders. We found a cortical lesion significantly reduced the RT success rate; however, a significant recovery was visible throughout the post-lesion rehabilitation period.

Moreover, using our new image processing method, we observed a significant deviation in the position of each of the five fingers relative to the target pellet in lesioned rats. Difficulties in forearm and shoulder movement probably caused these alterations. Our new method could be extremely beneficial for quantitative evaluations of RTs; it is also applicable to other types of brain-damaged rats. In the future, the current method should be compared with a more sophisticated evaluation system with a $3 \mathrm{D}$ analysis of forearm movements. The software used in the present study could be provided upon readers' requests.

\section{Conclusions}

The present study shows that the quantitative analysis method we have developed may play a critical role in evaluating the efficiency and dependability of new remedies for hand movement disorders. We found that the cortical lesion significantly reduced the RT success rate. however, a significant recovery was visible throughout the post-lesion rehabilitation period. Moreover, a significant deviation in the position of each of the five fingers, relative to the target food, was found in lesioned rats, using our new image processing method. These alterations are probably caused by the difficulties of the forearm and shoulder movements. Our new method could be extremely beneficial for RT quantitative evaluations; it is also applicable to other types of braindamaged rats.

\section{Author contributions}

YM performed data collection, analysis, and interpretation. $\mathrm{YO}$ and $\mathrm{AH}$ performed data collection and analysis. KI served as the research director. YM wrote the manuscript. All authors contributed to editorial changes in the manuscript. All authors read and approved the final manuscript.

\section{Ethics approval and consent to participate}

All experimental procedures were performed according to the National Institutes of Health guidelines for the care of experimental animals; the current experimental protocol was 
approved by the Institutional Animal Care and Use Committee at Maebashi Institute of Technology (A15-004).

\section{Acknowledgment}

The authors thank H. Mori and H. Komoto for their technical support. We also thank T. Kasamatsu for carefully reading the manuscript.

\section{Funding}

This work was supported in part by a Grant-in-Aid from the Japan Society for the Promotion of Science (JSPS KAKENHI Grant Number 19K19920) to YO.

\section{Conflict of interest}

The authors declare no conflict of interest.

\section{References}

[1] Brewer L, Horgan F, Hickey A, Williams D. Stroke rehabilitation: recent advances and future therapies. Quarterly Journal of Medicine. 2013; 106: 11-25.

[2] Lemon RN. Mechanisms of cortical control of hand function. The Neuroscientist. 1997; 3: 389-398

[3] Alaverdashvili M, Whishaw IQ. A behavioral method for identifying recovery and compensation: Hand use in a preclinical stroke model using the single pellet reaching task. Neuroscience \& Biobehavioral Reviews. 2013; 37: 950-967.

[4] Andrews AW, Bohannon RW. Distribution of muscle strength impairments following stroke. Clinical Rehabilitation. 2000; 14 79-87.

[5] Noskin O, Krakauer JW, Lazar RM, Festa JR, Handy C, O’Brien $\mathrm{KA}$, et al. Ipsilateral motor dysfunction from unilateral stroke: implications for the functional neuroanatomy of hemiparesis. Journal of Neurology, Neurosurgery \& Psychiatry. 2008; 79: 401-406.

[6] Sharpless SK. Reorganization of Function in the Nervous System-Use and Disuse. Annual Review of Physiology. 1964; 26: 357-388.

[7] Taub E, Pidikiti RD. Effects of motor restriction of an unimpaired upper extremity and training on improving functional tasks and altering brain behaviors. In Toole JF, Good DC (eds.) Imaging in Neurologic Rehabilitation (pp. 133-154). Demos Vermande: New York, NY. 1996.

[8] Jones TA. Motor compensation and its effects on neural reorganization after stroke. Nature Reviews Neuroscience. 2017; 18: $267-$ 280.

[9] Miltner WHR, Bauder H, Sommer M, Dettmers C, Taub E. Effects of Constraint-Induced Movement Therapy on Patients with Chronic Motor Deficits after Stroke: a replication. Stroke. 1999; 30: $586-592$.

[10] Gauthier LV, Taub E, Perkins C, Ortmann M, Mark VW, Uswatte G. Remodeling the Brain: plastic structural brain changes produced by different motor therapies after stroke. Stroke. 2008; 39 : 1520-1525.

[11] MacLellan CL, Gyawali S, Colbourne F. Skilled reaching impairments follow intrastriatal hemorrhagic stroke in rats. Behavioural Brain Research. 2006; 175: 82-89.

[12] Ramanathan D, Conner JM, Tuszynski MH. A form of motor cortical plasticity that correlates with recovery of function after brain injury. Proceedings of the National Academy of Sciences of the United States of America. 2006; 103: 11370-11375.

[13] Sacrey LA, Alaverdashvili M, Whishaw IQ. Similar hand shaping in reaching-for-food (skilled reaching) in rats and humans provides evidence of homology in release, collection, and manipulation movements. Behavioural Brain Research. 2009; 204: 153161

[14] Whishaw IQ, Pellis SM. The structure of skilled forelimb reaching in the rat: a proximally driven movement with a single distal rotatory component. Behavioural Brain Research. 1990; 41: 49-59.

[15] Whishaw IQ, Kolb B. The Behavior of the Laboratory Rat: A Handbook with Tests. Oxford University Press: Oxford, UK. 2005.

[16] Whishaw IQ, Whishaw P, Gorny B. The structure of skilled forelimb reaching in the rat: a movement rating scale. Journal of $\mathrm{Vi}$ sualized Experiments. 2008; 816.

[17] Whishaw IQ, Pellis SM, Gorny BP, Pellis VC. The impairments in reaching and the movements of compensation in rats with motor cortex lesions: an endpoint, video-recording, and movement notation analysis. Behavioural Brain Research. 1991; 42: 77-91.

[18] Whishaw IQ. Loss of the innate cortical engram for action patterns used in skilled reaching and the development of behaviora compensation following motor cortex lesions in the rat. Neuropharmacology. 2000; 39: 788-805.

[19] Kolb B, Cioe J, Whishaw IQ. Is there an optimal age for recovery from motor cortex lesions? I. Behavioral and anatomical sequelae of bilateral motor cortex lesions in rats on postnatal days 1,10 , and in adulthood. Brain Research. 2000; 882: 62-74.

[20] Gonzalez CL, Gharbawie OA, Williams PT, Kleim JA, Kolb B, Whishaw IQ. Evidence for bilateral control of skilled movements: ipsilateral skilled forelimb reaching deficits and functional recovery in rats follow motor cortex and lateral frontal cortex lesions. The European Journal of Neuroscience. 2004; 20: 3442-3452.

[21] Barth TM, Jones TA, Schallert T. Functional subdivisions of the rat somatic sensorimotor cortex. Behavioural Brain Research. 1990; 39: 73-95.

22] Biernaskie J, Chernenko G, Corbett D. Efficacy of Rehabilitative Experience Declines with Time after Focal Ischemic Brain Injury. Journal of Neuroscience. 2004; 24: 1245-1254.

[23] Erickson CA, Gharbawie OA, Whishaw IQ. Attempt-dependent decrease in skilled reaching characterizes the acute postsurgical period following a forelimb motor cortex lesion: an experimental demonstration of learned nonuse in the rat. Behavioural Brain Research. 2007; 179: 208-218.

[24] Gharbawie OA, Karl JM. Whishaw IQ. Recovery of skilled reaching following motor cortex stroke: do residual corticofugal fibers mediate compensatory recovery? European Journal of Neuroscience. 2007; 26: 3309-3327.

[25] Bova A, Kernodle K, Mulligan K, Leventhal D. Automated rat single-pellet reaching with 3-dimensional reconstruction of paw and digit trajectories. Journal of Visualized Experiments. 2019; $10.3791 / 59979$

[26] Knielinga M, MetzabI GA, Antonow-Schlorkea I, Wittea OW. Enriched environment promotes efficiency of compensatory movements after cerebral ischemia in rats. Neuroscience. 2009; 163: 759-769.

[27] Whishaw IQ, Dringenberg HC, Pellis SM. Spontaneous forelimb grasping in free feeding by rats: motor cortex aids limb and digit positioning. Behavioural Brain Research. 1992; 48: 113-125.

[28] van Lieshout ECC, Boonzaier J, Pel AJ, van Heijningen CL, Vink JJ, Visser-Meily JMA, et al. Translational Value of Skilled Reaching Assessment in Clinical and Preclinical Studies on Motor Recovery after Stroke. Neurorehabilitation and Neural Repair. 2021; 35: 457-467.

[29] Metz GA, Antonow-Schlorke I, Witte OW. Motor improvements after focal cortical ischemia in adult rats are mediated by compensatory mechanisms. Behavioural Brain Research. 2005; 162: $71-$ 82 .

[30] Imamura K, Tanaka S, Ribot J, Kobayashi M, Yamamoto M, Nakadate $\mathrm{K}$, et al. Preservation of functional architecture in visual cortex of cats with experimentally induced hydrocephalus. European Journal of Neuroscience. 2006; 23: 2087-2098.

[31] Sebastianelli L, Versace V, Taylor A, Brigo F, Nothdurfter W, Saltuari L, et al. Functional reorganization after hemispherectomy in humans and animal models: what can we learn about the brain's resilience to extensive unilateral lesions? Brain Research Bulletin. 2017; 131: 156-167. 
[32] Biernaskie J, Szymanska A, Windle V, Corbett D. Bi-hemispheric contribution to functional motor recovery of the affected forelimb following focal ischemic brain injury in rats. European Journal of Neuroscience. 2005; 21: 989-999.

[33] Gharbawie OA, Gonzalez CL, Whishaw IQ. Skilled reaching impairments from the lateral frontal cortex component of middle cerebral artery stroke: a qualitative and quantitative comparison to focal motor cortex lesions in rats. Behavioural Brain Research. 2005; 156: 125-137.
[34] Alaverdashvili M, Whishaw IQ. Compensation aids skilled reaching in aging and in recovery from forelimb motor cortex stroke in the rat. Neuroscience. 2010; 167: 21-30.

[35] Alaverdashvili M, Whishaw IQ. Motor cortex stroke impairs individual digit movement in skilled reaching by the rat. The European Journal of Neuroscience. 2008; 28: 311-322.

[36] Bury SD, Plautz EJ, Liu W, Quaney BM, Luchies CW, Maletsky RA, et al. A novel device to measure power grip forces in squirrel monkeys. Journal of Neuroscience Methods. 2009; 179: 264-270. 